Psychother Psychosom 2013;82:344-345

DOI: $10.1159 / 000348585$

\title{
Ifenprodil for the Treatment of Flashbacks in Adolescent Female Posttraumatic Stress Disorder Patients with a History of Abuse
}

\author{
Tsuyoshi Sasaki ${ }^{a}$, Kenji Hashimoto ${ }^{d}$,Keiko Okawada ${ }^{a}$, \\ Junko Tone ${ }^{a}$, Akira Machizawa ${ }^{b}$, Aya Tano ${ }^{a}$, Michiko Nakazatoc, \\ Masaomi lyo ${ }^{a, b, d}$ \\ Departments of a Child Psychiatry and ${ }^{\mathrm{b}}$ Psychiatry, and \\ ${ }^{\mathrm{C}}$ Research Center for Child Mental Development, Chiba \\ University Graduate School of Medicine, and d Division of Clinical \\ Neuroscience, Chiba University Center for Forensic Mental \\ Health, Inohana Campus, Chiba, Japan
}

A recent meta-analysis study showed that a history of abuse is associated with an increased risk for a lifetime diagnosis of multiple psychiatric disorders, such as posttraumatic stress disorder (PTSD), anxiety disorders, depression, eating disorders, sleep disorders, and suicide attempts [1]. In particular, PTSD is highly prevalent among women with a history of childhood abuse. Reexperiencing the event through intrusive flashbacks is one of the key diagnostic criteria for PTSD using ICD-10 [2], although the precise mechanisms for flashbacks are currently unknown [3]. Several lines of evidence suggest that glutamatergic neurotransmission via the N-methyl-D-aspartate (NMDA) receptor plays a role in certain behavioral manifestations common to PTSD, including dissociation and perceptual alterations $[4,5]$. There are currently no standard therapeutic agents for treating flashbacks associated with PTSD.

Ifenprodil (brand name Cerocral), a neuroprotective agent that binds to the GluN2B subunit of the NMDA receptor [6], is used as a cerebral vasodilator in a limited number of countries including Japan and France. Here, we report on 3 cases where ifenprodil proved effective in treating the flashbacks of adolescent female PTSD patients with a history of abuse. Written informed consent was obtained from the patients and their parents for publication of this case report.

Ms. A. was a 16-year-old Japanese female diagnosed with PTSD (F43.1) and other depressive episodes (F32.8), according to ICD10 criteria [2]. In junior high school, she was physically and sexually assaulted by a classmate for approximately 6 months. She subsequently suffered repetitive flashbacks, depressive episodes and irritation. Treatment with ifenprodil $(20 \mathrm{mg})$ was initiated, and after 3 weeks, the number of flashbacks was reduced by more than $60 \%$. The frequency of her flashbacks changed from 'frequently' (4) to 'rarely' (2), using the Likert five-point frequency scale. No side effects were reported in this patient.

\section{KARGER}

E-Mail karger@karger.com www.karger.com/pps
(C) 2013 S. Karger AG, Basel

This is an Open Access article licensed under the terms of the Creative Commons Attribution-NonCommercial 3.0 Unported license (CC BY-NC) (www.karger.com/OA-license), applicable to the online version of the article only. Distribution permitted for non-commercial purposes only.
Ms. B. was a 17-year-old Japanese female diagnosed with PTSD (F43.1) and other bipolar affective episodes (F31.8), according to ICD-10 criteria [2]. In junior high school, classmates physically assaulted her for approximately 2 months. She subsequently suffered repetitive flashbacks, depressive episodes and irritation. Treatment with blonanserin $(8 \mathrm{mg})$ and fluvoxamine $(50 \mathrm{mg}$ ) failed to reduce the incidence of flashbacks. New treatment with ifenprodil $(20 \mathrm{mg})$ was initiated and, after 2 weeks, her flashbacks were markedly reduced by more than $80 \%$. The frequency fell from 'very frequently' (5) to 'rarely' (2) using the Likert scale. Ms. B. also showed slight improvement in her dissociative symptoms. Her only reported side effect was a mild headache.

Ms. C. was a 19-year-old Japanese female, diagnosed with PTSD (F43.1) and other bipolar affective episodes (F31.8), according to ICD-10 criteria [2]. In elementary school, she was sexually assaulted by her uncle. She suffered flashbacks, depressive episodes and irritation for more than 10 years. She was treated with several drugs, including fluvoxamine, carbamazepine, valproate sodium, risperidone, blonanserin, quetiapine, and aripiprazole, and underwent psychotherapy, but none were effective in treating her flashbacks. Ifenprodil ( $20 \mathrm{mg}$ ) was added to the regimen and after 2 weeks, the number of flashbacks was reduced by more than $50 \%$. Flashback frequency fell from 'very frequently' (5) to 'occasionally' (3), using the Likert scale. In this patient, the only reported side effect was nausea.

None of these cases had a history of phencyclidine, ketamine, methoxetamine or tiletamine use. Nor was there use of any other recreational drug known to block glutamatergic neurotransmission via the NMDA receptor.

Ifenprodil was well tolerated in these 3 PTSD patients with histories of abuse. The depressive symptoms and irritation in 2 patients were first treated with antidepressants or atypical antipsychotics. Although their depression and irritation improved, the occurrence of flashbacks remained unchanged. Treatment with ifenprodil dramatically reduced the incidence of flashbacks in all patients. To our knowledge, this is the first report demonstrating the beneficial effect of ifenprodil for treating flashbacks in adolescent female subjects. Recently, Kishimoto et al. [7] reported that ifenprodil showed beneficial effects in the treatment of flashbacks, in female PTSD patients with a history of childhood sexual abuse. However, the precise mechanisms underlying this effect are unclear. It is also reported that ifenprodil is a potent agonist at endoplasmic reticulum chaperone sigma-1 receptors, which play a role in neuronal plasticity in the brain $[8,9]$. With its high affinity for both the NMDA and sigma-1 receptors, it is likely that ifenprodil acts, at least partially on these receptors, to alleviate flashbacks in PTSD patients, although further detailed studies are needed [7]. While selective serotonin reuptake inhibitors are effective in treating PTSD in children, adolescents and young adults [10], there is an increased risk of attempted suicide [11]. In contrast, there are no reports of an increased suicide risk in patients using ifenprodil.
Dr. Sasaki

Department of Child Psychiatry

Chiba University Graduate School of Medicine

Inohana 1-8-1, Chiba 260-8670 (Japan)

E-Mail sasaki@faculty.chiba-u.jp 
It seems likely therefore, that ifenprodil may be an effective and safe drug for the treatment of flashbacks associated with PTSD in children and adolescents.

In conclusion, ifenprodil therapy may prove to be an effective alternative treatment for flashbacks in adolescent female patients with PTSD, since this drug is already in clinical use. Nonetheless, more detailed randomized, double-blind studies are needed to confirm its efficacy.

The authors report no biomedical or financial interests, or potential conflicts of interest.

\section{References}

1 Chen LP, Murad MH, Paras ML, Colbenson KM, Sattler AL, Goranson EN, et al: Sexual abuse and lifetime diagnosis of psychiatric disorders: systematic review and meta-analysis. Mayo Clin Proc 2010;85:618-629.

2 World Health Organization: The ICD-10 Classification of Mental and Behavioural Disorders: Clinical Descriptions and Diagnostic Guidelines.

3 Maniglio R: The impact of child sexual abuse on health: a systematic review of reviews. Clin Psychol Rev 2009;29:647-657.
4 Chambers RA, Bremner JD, Moghaddam B, Southwick SM, Charney DS, Krystal JH: Glutamate and post-traumatic stress disorder: toward a psychobiology of dissociation. Semin Clin Neuropsychiatry 1999;4:274-281.

5 Cortese BM, Phan KL: The role of glutamate in anxiety and related disorders. CNS Spectr 2005;10:820-830.

6 Williams K: Ifenprodil, a novel NMDA receptor antagonist: site and mechanisms of action. Curr Drug Targets 2001;2:285-298.

7 Kishimoto A, Kaneko M, Gotoh Y, Hashimoto K: Ifenprodil for the treatment of flashbacks in female posttraumatic stress disorder patients with a history of childhood sexual abuse. Biol Psychiatry 2012;71:e7-e8.

$>8$ Hashimoto K, London ED: Further characterization of $\left[{ }^{3} \mathrm{H}\right]$ ifenprodil binding to sigma receptors in rat brain. Eur J Pharmacol 1993;236:159163.

-9 Ishima T, Hashimoto K: Potentiation of nerve growth factor-induced neurite outgrowth in PC12 cells by ifenprodil: role of sigma-1 receptor and $\mathrm{IP}_{3}$ receptor. PLoS One 2012;7:e37989.

10 Asnis GM, Kohn SR, Henderson M, Brown NL: SSRIs versus non-SSRIs in post-traumatic stress disorder: an update with recommendations. Drugs 2004;64:383-404.

11 Tandt H, Audenaert K, van Heeringen C: SSRIs (selective serotonin reuptake inhibitors) and suicidality in adults, adolescents and children. Tijdschr Psychiatr 2009;51:387-393. 